\title{
Food insecurity and malnutrition in Chinese elementary school students
}

\author{
Xiuhua Shen ${ }^{1,2}$, Xiang Gao ${ }^{3 *}$, Wenjing Tang ${ }^{1,2}$, Xuanxia Mao ${ }^{1,2}$, Jingyan Huang ${ }^{4}$ and Wei Cai ${ }^{1,2 *}$ \\ ${ }^{1}$ Clinical Nutrition Center, Shanghai Xin Hua Hospital, Shanghai 200092, China \\ ${ }^{2}$ Department of Nutrition, School of Medicine, Shanghai Jiao Tong University, Shanghai 200092, China \\ ${ }^{3}$ Department of Nutritional Sciences, Pennsylvania State University, University Park, PA 16802, USA \\ ${ }^{4}$ Project HOPE, Shanghai 200127, China
}

(Submitted 10 September 2014 - Final revision received 2 June 2015 - Accepted 22 June 2015)

\section{Abstract}

It has been shown that food insecurity is associated with poor diet quality and unfavourable health outcomes. However, little is known about the potential effects of food insecurity on the overall malnutrition status among children. In this study, we investigated the prevalence of food insecurity among 1583 elementary school students, aged 6-14 years, living in Chinese rural areas and examined its association with four malnutrition signs, including rickets sequelae, anaemia, stunting and wasting. Information on food security was collected via questionnaires. Rickets sequelae were assessed by an experienced paediatrician during the interview. Anaemia was determined by the WHO Hb thresholds adjusted by the local altitude. Weight and height were measured during the interview. Stunting and wasting were then evaluated according to WHO child growth standards (2007). We examined the association between food insecurity and the number of malnutrition signs (total number $=4$ ), and the likelihood of having severe malnutrition (presence of $3+$ signs), after adjusting for potential confounders, such as age, social-economic status and dietary intakes. During the previous 12 months, the overall prevalence of food insecurity was $6 \cdot 1 \%$ in the entire studied population and $16.3 \%$ in participants with severe malnutrition. Participants with food insecurity had a slightly higher number of malnutrition signs ( $1.14 v .0 .96 ; P=0.043)$ relative to those who were food secure, after adjusting for potential confounders. Food insecurity was also associated with increased likelihood of having severe malnutrition (adjusted OR 3.08; $95 \%$ CI 1.47, 6.46; P=0.003). In conclusion, food insecurity is significantly associated with malnutrition among Chinese children in this community.

\section{Key words: Food insecurity: Malnutrition: Children: Poverty}

Food insecurity (FIS) remains a leading public health problem in both developing and developed countries ${ }^{(1,2)}$. Previous studies have demonstrated that FIS may lead to inadequate dietary intakes and nutrient deficiencies ${ }^{(3-5)}$. These are particularly of concern for young children as they are highly susceptible to unsatisfactory food intakes ${ }^{(2,4)}$. However, the influence of household food insecurity (HFI) on school-aged children's growth and nutritional status remains understudied to date.

In a cross-sectional study including 670 adolescents (10-19 years) living in Tanzania, FIS was associated with undernutrition, as assessed by BMI for age ${ }^{(6)}$. HFI was also found to be associated with Fe-deficiency anaemia among participants aged 6-16 years in the National Health and Nutrition Examination Survey in the USA ${ }^{(3,7)}$. However, to our knowledge, there has been no study examining the relationship between HFI and other nutrient-deficiency signs, such as vitamin D-deficient rickets. Further, previous studies have generally focused on a single malnutrition symptom, and the effects of HFI on the overall malnutrition status, as assessed by diverse symptoms simultaneously, have not been examined.
China has been experiencing a transition from scarcity and extensive undernutrition to emerging nutrition-related non-communicable disease ${ }^{(8)}$. However, the nutritional improvement, along with the economic progress, is uneven in different areas in China. Many rural areas in central and western China are still experiencing poverty. To date, no populationbased FIS prevalence has been reported in China. Therefore, in the present study, we examined the FIS status in 1583 elementary school students living in poor mountain areas (Leye and Xundian) in southwest China and analysed its association with four malnutrition signs, including rickets sequelae, anaemia, stunting and wasting.

\section{Methods}

\section{Study population}

In 2011 the Chinese State Council Leading Group Office of Poverty Alleviation and Development stated that 592 povertystricken counties were being given priority in aid all over the

Abbreviations: FIS, food insecurity; HFI, household food insecurity.

* Corresponding authors: X. Gao, fax +1 814863 6103, email xxg14@psu.edu; W. Cai, fax +86 21 2507 6418, email caiw1978@163.com 
country by the updated standard of having an annual per-capita income $<2300$ yuan $^{(9)}$. Leye in Guangxi and Xundian in Yunnan are two of the 592 counties. Both counties are located in southwest China and characterised by high rocky mountains with low agricultural production of maize, rice and sugar cane

We conducted a school-based cross-sectional survey from October to December in 2011. A convenience sample of nine schools was selected from the two counties (six schools in Leye with 719 students and three schools in Xundian with 864 students). Two criteria were used to choose sample schools: (1) schools were listed as poor schools based on local government registration; (2) schools can be reached by motor vehicle. All school students from grades 1 to 5 were involved (6-14 years old, $47 \%$ were female) and participated in this survey. In total $99.6 \%$ of the eligible students participated. The field data collection was carried out by trained investigators (including one paediatrician, one nurse, one clinical test technician, two nutritionists and several senior medical students or interns). The protocols for this cross-sectional study were approved by the Ethical Review Committee at Xin Hua Hospital, affiliated to Shanghai Jiao Tong University School of Medicine.

\section{Assessment of household food insecurity}

The Chinese FIS questionnaire employed in the study was adapted from the general food sufficiency question/screener in the US Household Food-Security/Hunger Survey Module, which was developed by the United States Department of Agriculture (USDA) Food and Nutrition Service to measure FIS and hunger in the USA ${ }^{(10)}$. We selected this 'household food security' rather than 'child food security' questionnaire because all participants were boarding students. We made minor changes according to local habitual expression (online Supplementary Table S1). The first question was 'which of these statements best describes the food eaten in your household in the last 12 months?' Participants who selected 'enough to eat, with enough kinds of food (I/we) want to eat' (choice 1) or 'enough to eat, without enough kinds of food (I/we) want to eat often' (choice 2) were grouped as 'food sufficient', and if participants selected either 'sometimes not enough to eat or often not enough to eat' (choice 3) or 'often not enough to eat, and not enough kinds of food (I/we) want to eat' (choice 4), they were classified as 'food insufficient' and the follow-up question 'why people don't always have enough to eat' was asked. The household was classified as having 'FIS' if the respondent reported that the family either 'sometimes' or 'often' did not get enough food to eat because of not having enough money.

\section{Assessment of malnutrition}

Stunting and wasting. We measured the students' body weight and height by a calibrated, beam balance scale and stadiometer (Model RGZ-120; Changzhou Su Hong Medical Apparatus Factory) according to a standard protocol, and all of the participants were weighed with only one layer of undergarments and without shoes. The measurement was recorded to the nearest $0 \cdot 1 \mathrm{~kg}$ for weight and $0 \cdot 1 \mathrm{~cm}$ for height. Child $Z$-score height-for-age and $Z$-score BMI were computed using the WHO AnthroPlus software ${ }^{(11)}$. According to WHO standards ${ }^{(11)}$, stunting was defined as $Z$-score length-for-age $<-2$, and wasting was defined as $Z$-score BMI $<-2$.

Anaemia. We employed a standard measurement of blood $\mathrm{Hb}$ concentrations recommended by WHO for Fe-deficiency anaemia assessment in surveys, the cyanmethemoglobin method $^{(12)}$. Briefly, all participants underwent capillary blood collection by pricking the fingertip of the ring finger of the left hand, and $\mathrm{Hb}$ levels were measured with a type 721 spectrophotometer (Shanghai Precision Scientific Instrument Co. Ltd). The Hb cut-offs, $<115 \mathrm{~g} / 1$ for 5-11 years and <120 g/l for 12-16 years, recommended by WHO were used to diagnose anaemia $^{(12)}$. Because all studied schools were at altitudes of above $1000 \mathrm{~m}$, the original $\mathrm{Hb}$ criteria were adjusted by the formula of $\mathrm{Hb}_{\text {adjustment }}=\mathrm{Hb}_{\text {original criteria }} \times\left(1+\right.$ altitude in metres $\left./ 1000^{(12)}\right)$.

Rickets sequelae. The children were examined for hair depigmentation, abdominal distention and skin abnormalities by a paediatrician. Rickets sequelae (not rickets) were considered when the paediatrician observed one or more typical signs of rickets, including rachitic rosary, Harrison's groove, pigeon chest, enlarged wrists and bowed legs ${ }^{(13)}$.

\section{Assessment of covariates}

Dietary intake information was assessed by using a semi-quantitative FFQ and details have been published elsewhere ${ }^{(14)}$. Trained nutritionists completed the FFQ by asking the students to report their dietary consumption. We used the questionnaire to collect information on socioeconomic status, including parents' formal education, monthly income, occupation, the number of children in each family and household expenditure. Engel's coefficient was calculated from food expenditure divided by total expenditure in a family.

\section{Statistical analysis}

We used the Statistical Package for the Social Sciences for Windows (version 19.0; SPSS) for all statistical analyses. In order to capture the overall picture of the relationship between HFI and malnutrition, we used the number of four malnutrition signs (anaemia, rickets sequelae, stunting and wasting) as the primary outcome of the current analysis. We further examined each malnutrition sign individually. We also used the general linear model to test the relation between FIS status (FIS or no FIS) and the number of malnutrition signs. Logistic regression analysis was performed to study the association between food security status and the likelihood of having severe malnutrition (presence of $3+$ malnutrition signs) and each individual sign by calculating OR and their respective $95 \%$ CI. The potential confounders that may be associated with both food security or malnutrition were controlled in the models, including age, sex, consumption of egg, milk and meat, parents' 
income, educational level and occupation, the number of household children, Engel's coefficient and school. We also examined the interactions of FIS with age, sex, participant's school and Engel's coefficient, which may be an effect modifier for the association between FIS and malnutrition.

\section{Results}

During the previous 12 months, the overall prevalence of FIS was $6.1 \%$ in the entire studied population. The parents of children with HFI had lower educational level when compared with food secure (FS) families. FIS was also associated with lower household income $-87.0 \%$ ( $n$ 67) of father's and $90.0 \%$ ( $n$ 63) of mother's monthly incomes were $<800$ yuan (approximately $\$ 131$ ) in food insecure households, relative to $69.6 \%(n 725)$ and $81.5 \%(n 791)$, respectively, in food secure households. We also observed a significant positive association between FIS and more children $(2 \cdot 5$ ( $\mathrm{SE} 0 \cdot 1)$ in FIS $v .2 \cdot 2$ (SE $0 \cdot 1)$ in FS) in a family and being boarding students (Table 1 ).

The prevalence of malnutrition signs was high in this study; $16 \cdot 3 \%$ of the participants had severe malnutrition (i.e. having 3 or more malnutrition signs at the same time). Specifically, the prevalence of each individual malnutrition sign was $34.3 \%$ for stunting, $6.5 \%$ for wasting, $20.5 \%$ for anaemia and $36.0 \%$ for sequelae of rickets. Boys had higher prevalence of rickets sequelae than girls $\left(40.7 v .31 .0 \% ; P_{\text {difference }}<0.001\right)$. In contrast, we did not see significant sex differences for the other three malnutrition signs.

FIS was associated with the presence of more malnutrition signs (Figs 1 and 2). Participants with FIS still had a significantly higher number of malnutrition signs $(1.14 v .0 \cdot 96 ; P=0.043)$ relative to those who were food secure, after adjusting for potential confounding factors including age, sex, dietary consumption and several socio-economic status variables (e.g. parents' income and coefficient of Engel) (Table 2). Consistently, FIS was also significantly associated with higher likelihood (adjusted OR 3.08; 95\% CI 1.47, 6.46; $P=0.003$ ) of having severe malnutrition (Table 3 ). However, we did not observe a significant association between FIS and risk of having each individual malnutrition sign (Table 3). We did not observe significant interactions between FIS status and age, sex and Engel's Coefficient, in relation to malnutrition $\left(P_{\text {interaction }}>0.05\right.$ for all).

Except for FIS, food insufficiency (9.5\% of the studied population) was also significantly associated with higher numbers of malnutrition signs $(1.27 v .0 .87 ; P<0.001)$ and a higher likelihood (adjusted OR 2.84; $95 \%$ CI 1.47, 5.47; $P=0.002$ ) of having severe malnutrition, relative to those who were food sufficient, after adjusting for the afore-mentioned potential confounding factors.

\section{Discussion}

With the objective measurements of nutritional status, we observed that FIS is an independent risk factor for malnutrition among 6-14-year-old children and adolescents living in poor mountain areas in southwest China. Recent studies regarding childhood FIS conducted among children have
Table 1. Age- and sex-adjusted characteristics according to household food security status in 1583 elementary school students living in poor rural areas in southwest China*

(Mean values with their standard errors; percentages)

\begin{tabular}{|c|c|c|c|}
\hline & $\begin{array}{l}\text { Food security } \\
(n 1472)(\%)\end{array}$ & $\begin{array}{l}\text { Food insecurity } \\
(n 111)(\%)\end{array}$ & $P$ \\
\hline Age (years) & & & 0.62 \\
\hline Mean & $9 \cdot 8$ & $9 \cdot 7$ & \\
\hline SE & $2 \cdot 3$ & $2 \cdot 2$ & \\
\hline Female & $49 \cdot 1$ & 41.4 & 0.11 \\
\hline \multicolumn{4}{|l|}{ Ethnicity } \\
\hline Han & $72 \cdot 4$ & $58 \cdot 0$ & 0.01 \\
\hline Zhuang & $14 \cdot 2$ & $20 \cdot 0$ & \\
\hline Yao & $5 \cdot 4$ & $11 \cdot 0$ & \\
\hline Others & 8.0 & $11 \cdot 0$ & \\
\hline \multicolumn{4}{|l|}{ Egg consumption } \\
\hline Never & $9 \cdot 8$ & $15 \cdot 0$ & 0.44 \\
\hline$<1$ time/week & $73 \cdot 8$ & $70 \cdot 0$ & \\
\hline 1-2 times/week & $12 \cdot 3$ & $8 \cdot 0$ & \\
\hline >3 times/week & $4 \cdot 0$ & $7 \cdot 0$ & \\
\hline \multicolumn{4}{|l|}{ Milk consumption } \\
\hline Never & $29 \cdot 7$ & $37 \cdot 0$ & 0.06 \\
\hline$<1 \mathrm{bag} /$ week & $60 \cdot 8$ & $57 \cdot 0$ & \\
\hline 1-2 bags/week & 6.5 & $4 \cdot 0$ & \\
\hline >3 bags/week & $3 \cdot 1$ & $2 \cdot 0$ & \\
\hline \multicolumn{4}{|l|}{ Meat consumption } \\
\hline Never & $2 \cdot 6$ & $7 \cdot 1$ & 0.06 \\
\hline$<1$ time/week & $56 \cdot 9$ & $54 \cdot 5$ & \\
\hline 1-2 times/week & $22 \cdot 7$ & $26 \cdot 3$ & \\
\hline >3 times/week & $17 \cdot 9$ & $12 \cdot 1$ & \\
\hline \multicolumn{4}{|l|}{ Father's educational level } \\
\hline Illiterate & $8 \cdot 6$ & $23 \cdot 8$ & $<0.001$ \\
\hline Primary school & $54 \cdot 2$ & $55 \cdot 0$ & \\
\hline Middle school & 28.9 & $18 \cdot 8$ & \\
\hline >High school & $8 \cdot 3$ & 2.5 & \\
\hline \multicolumn{4}{|l|}{ Mother's educational level } \\
\hline Illiterate & 21.4 & $38 \cdot 8$ & $<0.001$ \\
\hline Primary school & $56 \cdot 8$ & $51 \cdot 3$ & \\
\hline Middle school & $17 \cdot 0$ & $8 \cdot 8$ & \\
\hline$>$ High school & $4 \cdot 8$ & $1 \cdot 3$ & \\
\hline \multicolumn{4}{|l|}{ Father's income } \\
\hline$<800$ yuan & $69 \cdot 6$ & $87 \cdot 0$ & 0.005 \\
\hline 800-1499 yuan & $19 \cdot 1$ & $7 \cdot 8$ & \\
\hline$>1500$ yuan & $11 \cdot 2$ & $5 \cdot 2$ & \\
\hline \multicolumn{4}{|l|}{ Mother's income } \\
\hline$<800$ yuan & $81 \cdot 5$ & $90 \cdot 0$ & 0.06 \\
\hline 800-1499 yuan & $12 \cdot 5$ & 8.6 & \\
\hline >1500 yuan & $6 \cdot 1$ & 1.4 & \\
\hline \multicolumn{4}{|l|}{ Father's occupation } \\
\hline Peasant & 74.4 & $77 \cdot 2$ & 0.61 \\
\hline \multicolumn{4}{|l|}{ Mother's occupation } \\
\hline Peasant & $78 \cdot 4$ & $78 \cdot 2$ & 0.84 \\
\hline Number of household children & & & $<0.001$ \\
\hline Mean & $2 \cdot 2$ & 2.5 & \\
\hline SE & 0.2 & $0 \cdot 1$ & \\
\hline Engel's coefficient & & & 0.75 \\
\hline Mean & 0.6 & 0.6 & \\
\hline SE & 0.0 & 0.1 & \\
\hline \multicolumn{4}{|l|}{ District } \\
\hline Leye, Guangxi & 91.7 & $8 \cdot 3$ & 0.07 \\
\hline Xundian, Yunnan & $94 \cdot 1$ & 5.9 & \\
\hline
\end{tabular}

shown that HFI could have unfavourable effects on childrens' mental, social and psycho-emotional development through different pathways ${ }^{(15-18)}$. Our results showed that the poor nutritional status of food insecure children could be one of the crucial pathways underlying these observed associations. 


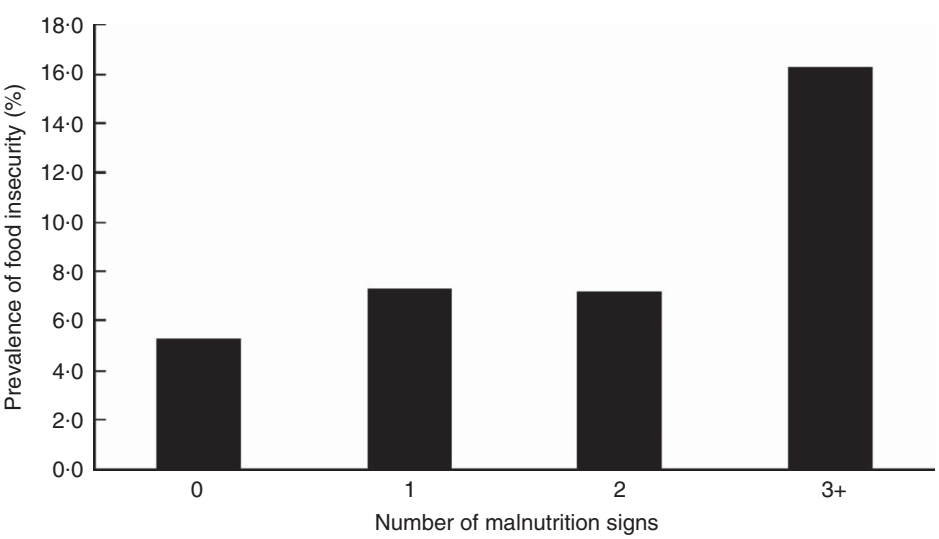

Fig. 1. Prevalence of food insecurity (\%) by number of malnutrition signs (total number $=4$; malnutrition signs include rickets sequelae, anaemia, stunting, wasting) among elementary school students in poor rural areas in southwest China.

Table 2. Number of malnutrition signs according to household food insecurity among elementary school students living in poor rural areas in southwest China*

(Mean values with their standard errors)

\begin{tabular}{|c|c|c|c|c|c|}
\hline & \multicolumn{2}{|c|}{$\begin{array}{l}\text { Food security } \\
\text { (n 1472) }\end{array}$} & \multicolumn{2}{|c|}{$\begin{array}{l}\text { Food insecurity } \\
\qquad(n 111)\end{array}$} & \multirow[b]{2}{*}{$P$} \\
\hline & Mean & SE & Mean & SE & \\
\hline \multicolumn{6}{|l|}{ Model $1 \dagger$} \\
\hline Number of malnutrition signs & 0.90 & 0.03 & $1 \cdot 20$ & 0.09 & 0.002 \\
\hline \multicolumn{6}{|l|}{ Model $2 \dagger$} \\
\hline Number of malnutrition signs & 0.91 & 0.03 & 1.23 & 0.09 & 0.001 \\
\hline \multicolumn{6}{|l|}{ Model $3 \dagger$} \\
\hline Number of malnutrition signs & 0.96 & 0.02 & 1.14 & 0.08 & 0.043 \\
\hline
\end{tabular}

Unlike three previous studies reporting the association between FIS and malnutrition (measured by BMI for age ${ }^{(6)}$ or blood Fe concentration ${ }^{(3,7)}$ ) among school-aged children or adolescents, our study employed a comprehensive and objective assessment of malnutrition. We used the number of malnutrition signs as an indicator, which combines all four signs of malnutrition into one and thus may reveal more meaningful relationships between FIS and overall nutrition status. Our findings suggest that the overall pattern of malnutrition may be more sensitive than any single malnutrition measurement. Considering the diversity of malnutrition signs, physical examination carried out by professionals is beneficial to diagnose certain nutrient-deficiency diseases. Therefore, besides the conventional measurement of malnutrition with weight, height and $\mathrm{Hb}$, a medical and physical check-up was performed by our experienced paediatrician, which revealed a high prevalence of rickets at the study sites.

The malnutrition outcomes related to FIS may be explained by insufficient food intake and poor diet quality. In these rural areas, approximately $80 \%$ of the families had only two meals per day. At the same time, eight out of nine studied schools provided only two meals per day for the boarding students. The students' average daily intake of energy, protein and Ca was
$6239.6 \mathrm{~kJ}(1491.29 \mathrm{kcal}), 36 \cdot 36 \mathrm{~g}$ and $163.14 \mathrm{mg}$, respectively, which were lower than the Chinese Recommended Nutrient Intake or Chinese Adequate Intake ${ }^{(19)}$. However, we still observed a significant association between FIS and malnutrition after adjusting for dietary intake, suggesting that FIS could be a potential risk factor for malnutrition, independent of dietary intake. Another potential explanation is the presence of residual confounding due to imperfect assessment of dietary intake. Another explanation is that other biological mechanisms could also be involved, which need to be elucidated in further studies. Because all participants were boarding students, we collected dietary intake information by interviewing these students, rather than their parents. We acknowledge that students might not be able to report their dietary intake accurately, which would inevitably introduce misclassification for dietary assessment. For the same reason, we did not employ the 'child FIS' questionnaire, which can provide more information but must be answered by parents.

The WHO growth reference for school-aged children was used in this study. Although this reference was developed on the basis of a multicentre study with 22917 representative samples from forty-five countries including China and has been widely used all over the world ${ }^{(20)}$, it has not been validated 
specifically for school-aged children living in rural areas. In this context, relevant results should be interpreted with caution. However, a recent study in which the China growth charts were compared with the WHO growth standards in assessing malnutrition of children under 5 years of age suggested that the WHO growth standards could be more applicable for international comparison of Chinese children ${ }^{(21)}$.

It is worth noting that we employed a simple version of the food security questionnaire (i.e. the general food sufficiency question/screener), rather than the full questionnaire standardised by the USDA, which has been employed in most US-based studies regarding food security ${ }^{(10)}$. This standardised scale may validly reflect the FIS experience of households in the USA and other countries ${ }^{(22)}$. Question 1 of this full questionnaire (i.e. the preliminary screener used in the current study) can provide a single-question measure that has a long history of use in surveys ${ }^{(10)}$. It has been reported that use of screener reduces the total burden in a survey and has merely low-to-modest effects on the loss of sensitivity in identifying food insecure households ${ }^{(10,11)}$

Another limitation is that the Chinese food security questionnaire has not been validated. In this context, we need to interpret our findings with caution because of potential misclassification for FIS assessment, which could attenuate the true association between FIS and malnutrition risk.

This study was also limited by its cross-sectional nature, which makes it impossible to establish causality. For example, we found that FIS was significantly associated with the overall pattern of malnutrition, but we did not observe a significant association between FIS and rickets alone. Because rickets generally occurs at the age range of 1-2 years, prospective cohort studies initiated in early childhood may help us to understand whether FIS in participants' early life could result in rickets. At the school age, like our study population, what we observed is generally sequelae of rickets rather than 'rickets' ${ }^{(13)}$. Similarly, stunting and wasting are also longstanding health outcomes, and the current study was not able to find causality between FIS and these conditions. Furthermore, the studied population is a convenience sample, which limited the generalisability of our findings. However, relative to the general population, this homogeneous sample is less confounded by socio-economic status, which could be important for studying the relationship between FIS and malnutrition. Although a cross-sectional analysis such as this cannot determine causality, it does identify the association between socio-economic status and FIS. We found that boarding students were more likely to experience FIS than were day students. The construction of boarding schools is one of the Chinese government's educational policies in order to integrate educational resources (teachers, facilities and buildings) to improve education quality in poor rural areas. Boarding students in the studied area are those who live in school during weekdays and return home during the school holidays (e.g. weekends). These students were not from economically privileged homes, as suggested by their economic status shown in Table 1. Boarding students may not be taken care of well by their parents. Therefore, it is essential for policymakers to make special efforts to reduce FIS in boarding schools, which might eventually contribute to a 
(a)

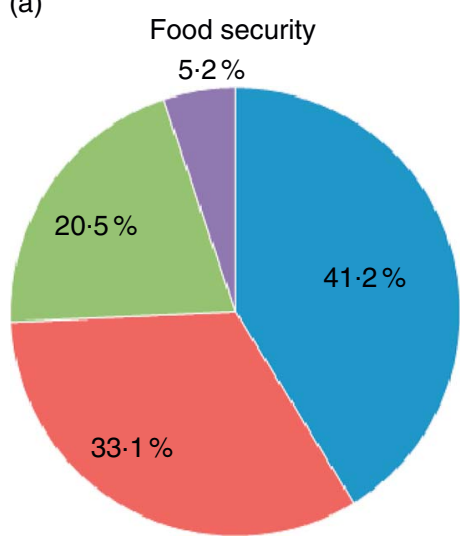

(b)

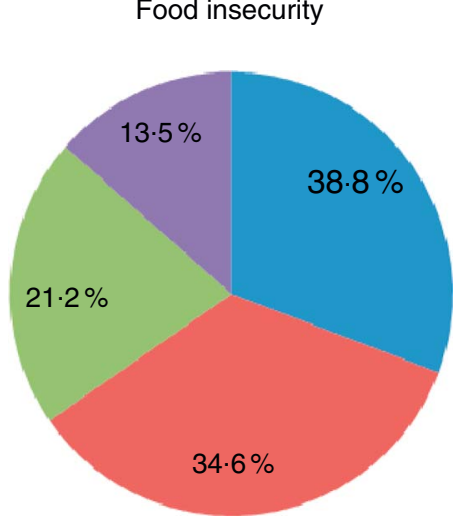

Fig. 2. Percentage of children with different numbers of malnutrition signs (total number =4; malnutrition signs include rickets sequelae, anaemia, stunting and wasting) according to their food security status ((a) food security; (b) food insecurity). , , 0 signs; , , sign; , 2 signs; , 3-4 signs.

reduction in the costs of treating the associated medical problems. On the other hand, boarding school policy provides convenient conditions for academics' and practitioners' professional assistance or nutritional intervention. Ultimately, all research on food security should contribute to improving the overall well-being of households and individuals by improving their diet quality ${ }^{(23)}$.

\section{Supplementary material}

For supplementary material/s referred to in this article, please visit http://dx.doi.org/doi:10.1017/S0007114515002676

\section{Acknowledgements}

The authors thank Dr Xie Qi and his research team in Nanxishan Hospital in Guilin, Guangxi, and Dr Li Meirui and her research team in Kunming Children's Hospital in Kunming, Yunnan, for their excellent, professional cooperation in the field data collection work.

This work was supported by Project HOPE 'The Abbott Fund Institute of Nutrition Science (AFINS)' program grant AFINSHOPE-2011-02.

The authors' responsibilities were as follows: X. S. designed and conducted research, analysed data and wrote manuscript; X. G. analysed data and wrote the manuscript; W. T. conducted research, collected and analysed data; X. M. and J. H. collected data; W. C. designed study. X. S., X. G. and W. C. had primary responsibility for final content. All authors read and approved the final manuscript.

There are no conflicts of interest.

\section{References}

1. Alisha C-J, Nord M \& Singh A (2013) Household Food Security in the United States in 2012. Washington, DC: Economic Research Service, USDA.

2. Ali D, Saha KK, Nguyen PH, et al. (2013) Household food insecurity is associated with higher child undernutrition in Bangladesh, Ethiopia, and Vietnam, but the effect is not mediated by child dietary diversity. J Nutr 143, 2015-2021.

3. Alaimo K, Olson CM, Frongillo EA Jr, et al. (2001) Food insufficiency, family income, and health in US preschool and school-aged children. Am J Public Health 91, 781-786.

4. Kaiser LL, Melgar-Quinonez HR, Lamp CL, et al. (2002) Food security and nutritional outcomes of preschool-age MexicanAmerican children. J Am Diet Assoc 102, 924-929.

5. Rose D \& Oliveira V (1997) Nutrient intakes of individuals from food-insufficient households in the United States. $\mathrm{Am} \mathrm{J}$ Public Health 87, 1956-1961.

6. Cordeiro LS, Wilde PE, Semu H, et al. (2012) Household food security is inversely associated with undernutrition among adolescents from Kilosa, Tanzania. J Nutr 142, 1741-1747.

7. Eicher-Miller HA, Mason AC, Weaver CM, et al. (2009) Food insecurity is associated with iron deficiency anemia in US adolescents. Am J Clin Nutr 90, 1358-1371.

8. Du SF, Wang HJ, Zhang B, et al. (2014) China in the period of transition from scarcity and extensive undernutrition to emerging nutrition-related non-communicable diseases, 1949-1992. Obes Rev 15, Suppl. 1, 8-15.

9. The Central Government of the People's Republic of China (2011) The standard for poverty alleviation and list of poverty-stricken counties http://www.cpad.gov.cn/ publicfiles/business/htmlfiles/FPB/fpqy/list.html (accessed 21 March 2014).

10. Gary B, Nord M, Price C, et al. (2000) Guide to Measuring Household Food Security, Revised 2000. Alexandria, VA: Food and Nutrition Service, USDA.

11. World Health Organization (2009) WHO AnthroPlus for Personal Computers Manual: Software for Accessing Growth of World's Children's and Adolescents. Geneva: WHO.

12. WHO, UNICEF \& UNU (2001) World Health Organization. Iron Deficiency Anaemia: Assessment, Prevention and Control, a Guide for Programme Managers. Geneva: WHO.

13. Strand MA, Perry J, Jin M, et al. (2007) Diagnosis of rickets and reassessment of prevalence among rural children in northern China. Pediatr Int 49, 202-209.

14. Shen X, Tang W, Mao X, et al. (2013) A questionnaire-based survey of dietary conditions of primary school students in poor mountain area of Guangxi and Yunnan. Chinese Journal of School Health 34, 3.

15. Alaimo K, Olson CM \& Frongillo EA Jr (2001) Food insufficiency and American school-aged children's cognitive, 
academic, and psychosocial development. Pediatrics 108 $44-53$.

16. Gundersen C (2013) Food insecurity is an ongoing national concern. Adv Nutr 4, 36-41.

17. Jyoti DF, Frongillo EA \& Jones SJ (2005) Food insecurity affects school children's academic performance, weight gain, and social skills. J Nutr 135, 2831-2839.

18. Kursmark M \& Weitzman M (2009) Recent findings concerning childhood food insecurity. Curr Opin Clin Nutr Metab Care 12, 310-316.

19. Tang W, Shen X, Mao X, et al. (2013) Investigation of nutritional status of pupils and dietary survey in primary schools in poor counties of Guangxi and Yunnan. Wei Sheng Yan Jiu 42, 571-575.
20. De Onis M, Onyango AW, Borghi E, et al. (2007) Development of a WHO growth reference for school-aged children and adolescents. Bull World Health Organ 85, 660-667.

21. Yang Z, Duan Y, Ma G, et al. (2015) Comparison of the China growth charts with the WHO growth standards in assessing malnutrition of children. BMJ Open, 25 February 2015.

22. Coates J, Frongillo EA, Rogers BL, et al. (2006) Commonalities in the experience of household food insecurity across cultures: what are measures missing? J Nutr 136, 1438S-1448S.

23. Perez-Escamilla R (2012) Can experience-based household food security scales help improve food security governance? Glob Food Sec 1, 120-125. 